\title{
Nurses' Experiences and Perceptions of Hourly Rounding: A Private Australian Catholic Hospital Single Case Study
}

\author{
Karen Francis, RN, PhD, MEd, MHIthScPHC ${ }^{1}$, Andriy Kurtsev, $R N^{2}$, Donna Walter, $R N^{3}$, \\ Cara Steele, BBSc, PGDPsy, PGDip, Stats ${ }^{4}$ and Carolyn Staines, BSc, PhD ${ }^{5 *}$
}

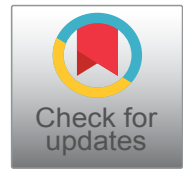

${ }^{1}$ Professor of Nursing, School of Nursing, Midwifery and Paramedicine, Australian Catholic University, Australia

${ }^{2}$ Nurse Unit Manager, St John of God Health Care, Australia

${ }^{3}$ Director of Nursing, St John of God Health Care, Australia

${ }^{4}$ Research Assistant, School of Nursing, Midwifery and Paramedicine, Australian Catholic University, Australia

${ }^{5}$ Research Project Manager, School of Nursing, Midwifery and Paramedicine, Australian Catholic University, Australia

*Corresponding author: Dr. Carolyn Staines, BSc, PhD, Research Project Manager, School of Nursing, Midwifery and Paramedicine, Australian Catholic University, 1200 Mair Street, Ballarat, VIC 3350, Australia

\begin{abstract}
Aim: This research paper reports on nurses' experiences and perceptions of hourly rounding undertaken at private Catholic acute care regional hospital in Australia.

Background: Evidence suggests that structured nursing rounds are associated with positive outcomes. However, a number of barriers to effective hourly rounding have been reported. Rounding practices were implemented in a medical/surgical ward at a regional hospital in Australia, in 2014, aiming to reduce the risk of patient falls and enhance the patient experience.

Methods: A qualitative, exploratory descriptive single case study design was utilised. Fifteen (15) nurses participated in the study and data were generated using Hermeneutic phenomenological interview technique. Transcriptions of each interview were analysed using both content and thematic analysis approaches.
\end{abstract}

Results: The experiences and perceptions of the nurse participants involved in the study were found to centre on the following themes and subthemes:

1. Support for rounding practice

2. Barriers to rounding practice

a) High workload and time-pressure

b) Documentation
Conclusion: The implementation of rounding was perceived to be useful and overall supported by the participants. Relocating nursing documentation and reviewing the model of care were highlighted as recommendations to improve compliance and support ongoing sustainability of rounding.

\section{Keywords}

Hourly rounding, Nurse perceptions, Nurse experiences, Nursing documentation

\section{Introduction}

Nurses are central caregivers in hospital contexts. They have a pivotal role in caregiving, securing patient safety and diagnosing alternations to health status and intervening to prevent deterioration [1]. Meaningful engagement with patients by caregivers enables caregivers to assess physiological and emotional wellbeing and intervene when and if appropriate. A nursing practice that seeks to enhance nurses' interactions with patients and improve patient outcomes is hourly rounding $[2,3]$. Rounding involves regular surveillance of patients, and their immediate environment, to identify potential hazards and monitor patient health status to enhance the likelihood that deterioration will be identified and interventions initiated [4].

- Evidence suggests that structured nursing rounds

Citation: Francis K, Kurtsev A, Walter D, Steele C, Staines C (2019) Nurses' Experiences and Perceptions of Hourly Rounding: A Private Australian Catholic Hospital Single Case Study. Int Arch Nurs Health Care 5:125. doi.org/10.23937/2469-5823/1510125 
are associated with positive outcomes. Patients feel safe and confident about their care, there is a reduction in patients' use of call bells and adverse events such as falls, medication errors and patient deterioration are prevented $[5,6]$.

- However, a number of barriers to effective hourly rounding have been reported. A systematic review [7] reported that competing tasks and priorities, limited time, and the workload in medical-surgical units were considered to interfere with consistent hourly rounding. Further, staff thought that the documentation associated with rounding was irrelevant and a waste of time. Lack of staff engagement in the process, challenges of meeting specific patient needs and lack of staff education prior to implementation were also identified as barriers.

- Many researchers have commented that a significant challenge reported by nurses who have used hourly rounding is the additional burden on their time $[4,8]$. Listening to nurses concerns and providing opportunities for comment and recommendations is a commonly advocated step in any change process including the introduction of new approaches to nursing work such as rounding $[4,9]$.

- In 2014, rounding practices were implemented in a medical/surgical ward at a Catholic private acute care regional hospital in Victoria, Australia, with the aims of reducing the risk of patient falls and enhancing the patient experience. This study sought to understand the nurses' experiences and perceptions of rounding following this implementation, using a qualitative exploratory descriptive single case study design.

\section{Methodology}

In Australia, there are two levels of regulated nurses: Registered nurses (RNs), who are degree prepared and are the largest group, and enrolled nurses (ENs), who are diploma level educated [10]. The hospital and the ward involved in this study utilises a team nursing model of care $[11,12]$. The teams most often involved two nurses who were generally registered nurses. The teams were allocated patients to whom they deliver total patient care.
A qualitative exploratory descriptive single case study design was employed [13]. All registered and enrolled nurses working at the hospital who were implementing hourly rounding, during a 12-month implementation period, were eligible to participate in the study. Participation in the study was voluntary and time release from work for the study was accommodated.

Confidential individual interviews were conducted, using a Heideggerian hermeneutic phenomenological interview approach, over a five-week period in late 2015. They were based on a set of questions (Table 1 ) which, where appropriate, were supplemented with probing to further explore participants' responses or to seek clarification.

All interviews were digitally recorded and subsequently transcribed verbatim. The textual data generated from the interviews were examined using content and thematic analysis techniques. Each transcript was read several times in order to familiarise the reader with the text, followed by content analysis, producing an overview of the transcript, and line-by-line thematic analysis. This approach led to the distillation of key phrases and/or conceptualisations (codes) that reflected participants' experiences. These were further interrogated and like codes were clustered and given descriptive titles that reflected the theme. Finally, all analyses were compared, with like ideas grouped together under an existing code or a new code that better reflected the central theme [14-16]. Implicit and explicit ideas embedded in the texts were isolated, enabling the phenomenon of interest to be explicated: Nurses' experiences and perceptions of hourly rounding.

This study was been approved by the organisation's Human Research Ethics Committee.

\section{Findings}

A satisfactory level of participation was achieved in the study with $40 \%$ of nurses employed on the ward taking part. Fifteen (15) nurses were interviewed. Fourteen (14) of the interviews were conducted faceto-face, on-site at the hospital, in a quiet private space on the ward where the nurses worked. For logistical reasons, one (1) interview was conducted via telephone.

Table 1: Interview questions.

1. Can you tell me about your experience of 'rounding'?

2. What do you consider to be the strengths associated with incorporating 'rounding' into your practice?

3. Can you tell me about any challenges that you faced when using 'rounding'?

4. Can you tell me about any challenges that you faced when using 'rounding'?

5. Thinking about the whole nursing team can you tell me what you think the team feels about 'rounding' and why?

6. Are there any recommendations that you would make to:

a. Enhance practice;

b. Improve patient satisfaction and;

c. Reduce risks? 
Table 2: Characteristics of participants: Gender, qualifications, employment status, length of service.

\begin{tabular}{|l|l|}
\hline \multirow{2}{*}{ Gender } & Female: $13(86.7 \%)$ \\
\hline \multirow{2}{*}{ Qualifications } & Male: 2 (13.3\%) \\
\hline \multirow{2}{*}{ Employment status } & Registered nurse: $14(93.3 \%)$ \\
\hline & Enrolled nurse: $1(6.7 \%)$ \\
\hline & 0.7 to 0.9 EFT: $13.3 \%$ \\
\hline \multirow{2}{*}{ Length of service } & $<0.7$ EFT: $33.3 \%$ \\
\hline & Range: $1-20$ years (mean = 5.2 years) \\
\hline & $<5$ years: $66.7 \%$ \\
\hline
\end{tabular}

Eight (8) interviews involved two researchers and seven (7) interviews were conducted by one researcher. The duration of interviews was, on average, 17 minutes (Mean $=16.44, \mathrm{SD}=5.04)$.

Participant characteristics are shown in Table 2.

The experiences and perceptions of the nurse participants involved in the study were found to centre on the following themes and subthemes:

1. Support for rounding practice

2. Barriers to rounding practice

a) High workload and time-pressure

b) Documentation

\section{Support for rounding practice}

The majority of participants felt that hourly rounding complemented and supported quality nursing care:

... the introduction of the tool was good in a way that it, sort of, prompted people to be more vigilant with what they are doing with their patients. (RN1)

Some participants reported that the patients appreciated the additional time nurses spent with them. When told that there would be hourly rounding some patients responded:

'Oh wow, that's great'... 'Oh wow, I've never been told that before - I thought you just came in when we buzzed'

For most participants, hourly rounding was a viewed as a team process. Knowing the patients and sharing information with the nursing team throughout each shift and across shifts was valued. Implementing rounding on a trial basis was thought to be a useful process.

Rounding was also considered to provide nurses with both peace of mind and evidence of care:

I try to get around and introduce myself and check on each patient throughout the course of the shift, but it's nice to actually have that peace of mind that each patient is being looked in on at least every hour.

So that it's not then possible for them to say 'well
I haven't had someone come in to me for the last four hours, I've been sitting in this bed and no one has actually been in to check on me. (RN1)

\section{Barriers to rounding practice}

High workload and time pressure: The busy nature of the ward was highlighted as a factor that impacted on participants' capacity to comply with the hourly rounding protocol. Participants explained that competing priorities, such as preparing patients for theatre, or monitoring patients on return from theatre, took precedence over other activities:

... a busy day on the surgical ward, and seeing that I've missed four or five roundings. Because I've just been so busy picking up patients or doing whatever else I have been doing. (RN2)

It was felt that the busyness of some shifts made it hard, if not impossible, to comply with the hourly rounding protocol:

I do it when I've got time. And I try and consciously do it. I do it more on an afternoon shift; on a morning shift not so much. (RN2)

Documentation: Much of the participants' concerns about hourly rounding focussed on the completion of the rounding documentation. The nurses were required to record each rounding visit on a chart. They needed to record the date, time of visit and the following patient status codes as appropriate: C (comfortable); T (toileting); L (leave/appointment); PAC (pressure area care); S (sleeping); R (refused); A (analgesia). The charts were stored in a locked drop-down box outside the patients' rooms, along with other paperwork.

All participants commented on the burden related to hourly rounding documentation. While participants accepted that completion of rounding documentation was required, time pressures meant that compliance was difficult:

I think it's a good idea, but it is not very practical ... We already do a lot of paperwork and ... you just can't do it. And it's not a priority, at all... if people come 'round and say 'oh you haven't been filling in it' and I think that is totally unreasonable, because you can't. (RN2)

I haven't had a chance to write it down; I'd like to but I don't want to fill it in just because. I don't want to just write 'yes they've been comfortable' and just tick-ticktick because I don't feel like that's giving accurate data from me actually doing it. (RN3)

Although I've been into the rooms I haven't actually had a chance to tick off my rounding. (RN2)

Some admitted that document completion was often retrospective:

... we get really busy and it's kind of put on the back burner a bit, like we probably don't fill it in, it's probably 
one of the last pieces of work to get done. (RN4)

One experienced registered nurse, while considering hourly rounding to be a useful approach '... [it was] drilled into us years ago - round on the patients every hour, it was just never recorded', reported finding the documentation challenging:

... I fall behind in it. I try to keep up with the time but then a couple hours can go by and you haven't written down the time. And I notice people doing it retrospectively. 'Oh yes I was in there at that time but didn't sign the form'. (RN5)

Some participants were concerned that failure to complete documentation would impact negatively on their reputation. One participant initially thought that hourly rounding was about checking up on the nurses and expressed concern about how others would perceive incomplete documentation:

... you get to the end of a shift and you see it's not documenting, so then I'm worried that whoever reads that, is now going to think well [name] hasn't been in there for five hours, which isn't the case, but that's just how it makes you feel sometimes. (RN4)

There was also concern that the documentation failed to capture the reality of the work load:

Sometimes you are actually with a patient more than ten times within the hour but you may see at the end of the day that within that hour there is no evidence that someone has ticked off to say l looked after them for one or two or three things. (RN1)

It was also thought that the predetermined categories of patient status did not reflect the reality for some:

... yesterday we had a patient pass away ... She was comfortable but she was also unconscious ... I don't know what else you would write other than 'comfortable' but this is where it gets, where we were all discussing it, ... I don't know what wording you would use because, she was dying and in a sense she was comfortable, and an hour later she's gone. (RN6)

She was also concerned that as the documentation was being used in a limited way and could fail to capture potentially important information. She provided the following scenario to highlight her anxiety about the current tool:

Probably the issue is that people aren't writing; they tend to be more just putting 'comfortable' 'asleep' or you know, that sort of thing,... I don't think it's quite capturing, it's not bad, but sometimes patients are in between 'comfortable' and 'analgesia' ... If the patient was agitated you would write 'agitated' ... we just need a bit more work around that

... At the end of the day those documents are lawful documents that can be subpoenaed so it is important that if something is out of the ordinary that people understand that there is an opportunity to maybe write something else. (RN6)

Difficulty of access to the rounding documentation was also thought to a barrier to compliance. Many participants commented that the documentation's location in a locked box outside the rooms was a problem:

Oh yes I was in there at that time but didn't sign the form, because the form is outside in the locked up box. (RN5)

One suggestion to overcome this was to use the whiteboards already in patients' rooms:

... we've got those boards in the room, the white boards, if you just had it on that, when you went in to do someone's obs you signed, you're in there, they're comfortable, easy. (RN2)

The location of the documentation in secured boxes was also seen as a barrier to coordination of team based care. It was felt that easier access to rounding information would enable team members to determine whether a patient visit was due and could avoid duplication:

... if we had like a rounding check on our planner it might be good and then I can 'oh my colleague's already been in there she's done that' 'cause sometimes you can go into a room and say, 'do you need to go the toilet' and they'll say, 'My colleague only asked me ten minutes ago'. So sometimes we can double up. (RN4)

However, concern about documentation was not unanimous. One night-duty nurse considered completion of the documentation to be easy. Although, he added that on night duty there is more time to focus on the documentation and therefore it is less burdensome than it is on day and evening shifts:

... you've got a bit more time to usually sit down and do it and make sure that you're doing it properly. Obviously you are not talking to the patient waking them up all the time but it's a good record of what happens overnight. (RN7)

This again suggests that the busyness of the unit may be a factor contributing the difficulties associated with documentation compliance.

\section{Implementation and management}

The nurses' experience of rounding, and their subsequent comments about it, occurred within the context of a 12-month implementation program. The following gives an overview of the features and challenges of implementation of rounding practice at the hospital.

Nature of workforce: The nursing workforce in Australia, and at this hospital, is predominantly parttime and there is a high degree of use of casual staff to meet workforce requirements. In this study, $86.6 \%$ 
of the nurses participating had part-time positions. This workforce structure has the potential to present problems for the implementation of practice changes. It would be difficult to ensure that all members of a large casual or part-time workforce are well informed about changes and are completely familiar with the practice protocol.

Change of documentation: The hospital found it necessary to change the nature of the rounding documentation during the period of implementation. The initial chart had a strong focus on hourly rounding and used a 24-hour clock image, with hourly segments, where nurses recorded their compliance with the rounding protocol. This method of charting was unfamiliar to the nurses and they raised their concerns about it with the ward management. Consequently, the chart was replaced with one that reflected the formatting of other nursing data collection forms such as care plans, observation charts and medication charting.

Education of nurses: Prior to implementation of the hourly rounding pilot, nursing staff participated in a professional development program to acquaint them with hourly rounding practice. However, not all nurses rostered to the ward were able to attend and there was no provision made for subsequently educating new staff members or casual nursing staff.

Monitoring of rounding performance: Rounding documentation was audited by the Nurse Unit Managers who provided feedback to nurses at handovers and at weekly staff meetings.

Change from hourly: While the initial rounding protocol implemented at the hospital required hourly rounding, over the course of the implementation this changed to a less frequent, more opportunity based routine. This change was unofficially initiated by the nurses who found they were unable to comply with the hourly protocol. While acknowledging that hourly rounding would be ideal, participants found they needed to modify their practice to accommodate the high workloads and competing demands they experienced.

\section{Discussion}

This study found that the rounding protocol was perceived to be useful and was overall supported by the participants. However, full compliance with the protocol was found to be challenging. Heavy workloads and the busyness of the wards made it difficult to achieve rounding visits on an hourly basis and the requirement to document the rounding visits was perceived to be a burden and considered to be of low priority. This perceived burden of the documentation was in line with other studies reporting concern about the documentation requirement associated with rounding practices $[17,18]$.

The introduction of any change, such as the adoption of rounding practices, requires organisational commitment and investment in developing and implementing a well-planned change process. This should include considered communication, education and training strategies $[19,20]$ that engage the staff who will be impacted [21]. In the Australian setting, where there is casualization of the workforce, and in the setting of this study where the majority of staff were part-time, ensuring adequate and consistent communication and education can be difficult.

Change processes must include opportunity for discussion throughout, and following, implementation. Listening and responding to end users is advocated and can be the deciding factor between successful or unsuccessful change processes [19]. The findings of this study suggest that hourly rounding should be modified to align with current work practices and the model of care used within the organisation in agreement with McLeod and Telzlaff [3].

These authors advocate ongoing consultation with nursing staff to identify facilitators and barriers that enhance and/or impede rounding. Furthermore, they suggest collaborative problem solving as a necessary technique to secure ongoing sustainability of this process. Such approaches could be the catalyst for staff development projects of the future.

Operationalizing the rounding practice protocol was difficult, however, and resulted in modifications being implemented that included moving from hourly to purposeful rounding. Relocating nursing documentation and reviewing the model of care were highlighted as recommendations to improve compliance and support ongoing sustainability of purposeful rounding.

\section{Limitations}

This study was undertaken to evaluate nurses' experiences of hourly rounding on a single ward of a private hospital. While there was a good level of participation in the study, with $40 \%$ of nurses employed on the ward taking part, the data collection was limited to only a single time point, i.e. following the completion of the 12-month implementation period. It is possible that capturing nurses' perceptions at a number of times points throughout the period may have revealed changes in perceptions, possibly providing additional insights to inform the effective implementation of rounding practices.

\section{Conclusion}

This study has presented an evaluation of nurses' experiences and perceptions of hourly rounding that was introduced on a ward of a private Australian Catholic regional hospital. The study findings indicated that nurses were supportive of the overall intent of hourly rounding but encountered problems operationalizing the practice protocol. The 'busyness' of the ward, and the model of 
care (team nursing with teams most often consisting of two nurses who were allocated patients for whom they provided all care), were impediments that led to nurses modifying the process of hourly rounding. In practice, this involved checking on patients routinely, as per the protocol, but failing to complete documentation when competing priorities took precedence. The positioning of the documentation outside patients' rooms was identified as a major obstacle to completing the associated paperwork following each rounding. The model of care, while not highlighted directly by participants, was indicated as incompatible with hourly rounding. To ensure that nurses' voices are heard, and patient care and safety maintained, regular authentic consultation and feedback must be a feature of the workplace.

\section{Ethics Statement}

This research was conducted with the approval of the Human Research Ethics Committee of St John of God Healthcare.

\section{References}

1. Kozier B, EG Lea, Berman A, Snyder S, Levett-Jones T (2015) Kozier and Erb's fundamentals of nursing. ( $3^{\text {rd }} \mathrm{edn}$ ).

2. Studer Group (2007) Hourly Rounding Supplement. Best Practice: Sacred Heart Hospital, Pensacola, Florida.

3. McLeod J, Telzlaff $S$ (2015) The value of purposeful rounding. American Nurse Today 10: 6-7.

4. Deitrick LM, Baker K, Paxton H, Flores M, Swavely D (2012) Hourly rounding: Challenges with implementation of an evidence-based process. J Nurs Care Qual 27: 13-19.

5. Hutchings M (2012) Caring around the clock: Rounding in practice. Nurs Times 108: 12-14.

6. Meade CM, Bursell AL, Ketelsen L (2006) Effects of nursing rounds: On patients' call light use, satisfaction, and safety. Am J Nurs 106: 58-70.

7. Toole N, Meluskey T, Hall N (2016) A systematic review: Barriers to hourly rounding. J Nurs Manag 24: 283-290.

8. National Nursing Research Unit (2012) Policy+ 35
Intentional rounding: What is the evidence? In: J Maben, Policy+ review. King's College London, London.

9. Kotter JB (2012) Leading change. Harvard Business School Press, USA.

10. Australian Nursing Federation (2012) Facts Sheet 2: A snapshot of nursing in Australia.

11. Fernandez R, Johnson M, Tran DT, Miranda C (2012) Models of care in nursing: A systematic review. Int $\mathrm{J}$ Evid Based Healthc 10: 324-337.

12. Kalisch B, Schoville R (2012) It takes a team. Am J Nurs 112: 50-54.

13. Stake RE (1995) The art of case study research. Sage Publications, Thousand Oaks, CA.

14. Harding T, Whitehead D (2016) Analysing data in qualitative research. In: DWZ Schneider, G LoBindo-Wood, J Haber, Nursing and Midwifery Research, methods and appraisal for evidenced-based practice (Australia and New Zealand edn), Chatswood Elsevier.

15. Taylor B, Kermode S, Roberts H (2006) Research in nursing and health care: Evidence for practice. ( $3^{\text {rd }}$ edn), Thomson, Australia.

16. Taylor B, Francis K (2013) Qualitative research in the health sciences: Methodologies, methods and processes. Routledge, London.

17. Neville K, Lake K, LeMunyon D, Paul D, Whitmore K (2012) Nurses' perception of patient rounding. J Nurs Adm 42: 8388.

18. Walker K, Duff J, Fitzgerald K (2014) 'Rounding' for better patient care: An evaluation of an improvement intervention implementation. Int J Nurs Pract 21: 207-213.

19. Dąbrowska E, Sielska J, Zdanowska J (2014) Change management in health care - overcoming mental and organisational barriers. Polish Nursing 54: 337-342.

20. Matos Marques Simoes P, M Esposito (2014) Improving change management: How communication nature influences resistance to change. Journal of Management Development 33: 324-341.

21. Fabry D (2015) Hourly rounding: Perspectives and perceptions of the frontline nursing staff. J Nurs Manag 23: 200-210. 